\title{
Flame temperature trends in reacting vanadium and tungsten ethoxide fluid sprays during $\mathrm{CO}_{2}$-laser pyrolysis
}

\author{
B.W. Mwakikunga • A.E. Mudau • N. Brink • \\ C.J. Willers
}

Received: 8 November 2010 / Revised version: 7 July 2011 / Published online: 10 September 2011

(c) The Author(s) 2011. This article is published with open access at Springerlink.com

\begin{abstract}
We observe the "invisible-to-the-naked-eye" flames of tungsten and vanadium ethoxide aerosols when ignited at moderate laser excitation $\left(0<P_{\text {laser }}<70 \mathrm{~W}\right)$ by employing an IR thermo-graphic camera. No emission is seen in the visible range whether by the visible region cameras or by spectroscopy. The emissivity of the precursor solution measured was 0.80 and 0.75 for tungsten and vanadium ethoxide, respectively. The spectral emissivities of the tungsten and vanadium ethoxide flames measured using FTIR-spectrometer were used to calculate the pyrolysis flame temperature at various laser intensities and wavelengths. New energy balance equations have been derivedthe transient temperature one extended from HaggertyCannon equation and the other based on standard resonance analysis. Fitting these models to experimental data reveals
\end{abstract}

B.W. Mwakikunga $(\bowtie)$

DST/CSIR National Centre for Nano-Structured Materials,

P.O. Box 395, Pretoria 001, South Africa

e-mail: bmwakikunga@csir.co.za

Fax: +27-12-8413152

B.W. Mwakikunga

CSIR National Laser Centre, P.O. Box 395, Pretoria 001,

South Africa

B.W. Mwakikunga

Department of Physics and Biochemical Sciences,

The Polytechnic, University of Malawi, Private Bag 303, Chichiri, Blantyre 3, Malawi

A.E. Mudau $\cdot$ C.J. Willers

CSIR Defence, Peace, Safety and Security (DPSS), P.O. Box 395, Pretoria 001, South Africa

N. Brink

DENEL Dynamics, Nelmapius Drive, Centurion, P.O. Box 7412, Centurion 0046, South Africa that only small amounts $(1.33 \%$ and $4.32 \%$, respectively) of the laser power are used in the pyrolysis of the precursor ethoxide aerosols into the desired oxide nanostructures. The low levels of specific heat capacity values obtained in these sprays suggest that these are electronic heat capacities rather than lattice heat capacities; enthalpies are also obtained. The experimental temperature-laser power trends observed were in agreement with previous findings from Tenegal et al. (Chem. Phys. Lett. 335:155, 2001). The damping coefficients, and hence the saturation intensities confirm that the vanadium containing precursor liquid is harder to dissociate into final products than the tungsten precursor as observed experimentally.

\section{Introduction}

The laser pyrolysis technique has gained popularity as a relatively new materials processing tool recently. The technique involves interaction between the laser beam (mainly the continuous wave $\mathrm{CO}_{2}$ laser at a wavelength of $10.6 \mu \mathrm{m}$ ) and the starting materials in a liquid spray form (aerosol). The spray and beam are made to interact orthogonally and the beam diameter, $w_{\text {laser }}$, and the aerosol spray diameter, $d_{\text {spray }}$, are controlled in such a way that $d_{\text {spray }}<w_{\text {laser }}$. This is to ensure that all the spray molecules are inside the beam for complete combustion. To date, there are about more than 100 publications on materials processed by this technique. While most of these reports have presented on the nanoproducts of laser pyrolysis, only a few have proposed explanations on the interaction between the laser beam and the aerosols. There have been conflicting conceptions about the nature of interaction between the laser beam and the fluid spray with two major schools of thought-photochemical theory and photothermal theory [1]. In this paper, we attempt 
to shed light on both theoretical advancements and empirical measurements of the temperature at the interaction zone as it depends on laser intensity and laser wavelength. A brief review is important for a background.

As early as 1873, Maxwell used the electromagnetic theory to calculate force on solid bodies due to absorption or reflection of light [2] and in the early 1900s quantitative measurement of the force on bodies and gases were done especially as well demonstrated by the Crooke's radiometer [3]. In 1917, Einstein [4], using the quantum theory to calculate the influence of the electromagnetic radiation on the motion of molecules, showed the importance of the Planck's blackbody spectrum in his calculation. In later years, 1933, Frisch performed the first experiment showing momentum transfer to atoms by photon absorption, and also in 1950 Kastler used light to cool and heat atoms. In the 1970s, with the advent of more intense lasers, more pronounced effects on deflection of atom beams were observed. The first laser pyrolysis experiments (named differently then) with the $\mathrm{CO}_{2}$ laser tuned at its $10 \mathrm{P}_{20}$ line of wavelength $10.59 \mu \mathrm{m}$ are known to have been first performed by Bachmann [5] between 1974 and 1975. Laser pyrolysis of vanadium and tungsten compounds, in our group, has led to very interesting nanostructures-nanowires of $\mathrm{WO}_{3}$ and nanobelts of $\mathrm{VO}_{2}$ [6-9]. These nanostructures were obtained without the knowledge of the temperature of processing as has been the case with many reports on laser pyrolysis. However, for repeatability, the knowledge of temperature of the aerosol molecules is absolutely important.

Measurement of this temperature cannot be accomplished with the traditional thermometer or thermocoupleany contact with the aerosol changes the temperature one is intending to measure. For this reason, noncontact (optical) methods are preferred. Various attempts to understanding the relationship between laser intensity and wavelength on the temperature and dissociation protocols are outlined. Since these theories have failed to explain some recent experimental data on laser intensity versus aerosol temperature, this paper presents new laser-intensity-aerosoltemperature and laser-wavelength-aerosol-temperature data. We also attempt to fill in the gap that exists in the explanation of such data.

\section{Brief review of the $\mathrm{cw} \mathrm{CO}_{2}$ laser interaction with sprayed fluids}

A theoretical treatment by Bowden et al. [10] in 1977 proposed a quantum-mechanical model for laser-induced photo-chemical reactions in which selective low-level excitation of a molecular species by the laser beam was assumed to incorporate coherent resonant energy transfer with collision damping. The resonant bonds were seen to be the heat sources and the nonresonant ones were the heat sinks. The Bowden et al. model was designed based on a pulsed $\mathrm{CO}_{2}$ laser source. In the case where the laser pulse width is much smaller than the molecule collision times, one has a collision-free interaction and vice versa. The Hamiltonian which Bowden et al. developed especially for the laser interaction with gas molecules with collisions and collision damping led them to an equation which gave the probability, $n$, of a molecule or atoms being excited when in the laser beam to depend on the resonant frequency, $\omega$, the laser detuning (or how far away from resonance the laser frequency is), $\Delta=\omega-\omega_{\text {laser }}=2 \pi\left[(1 / \lambda)-\left(1 / \lambda_{\text {laser }}\right)\right]$ and the intensity of the laser beam, $I_{\text {laser }}$ given as $n=(1 / Q)+\left(I_{\text {laser }} / S\right)$ where $Q=\exp \left(\hbar \omega / k_{B} T\right)-1$ and $S=\left(\Delta+\Omega_{R}\right)^{2}+\eta^{2}$. The symbol, $\Omega_{R}$, is the Rabi frequency defined as the frequency of a molecule or an atom flopping between excited and ground states, $\hbar=h / 2 \pi$ where $h$ is the Planck's constant and $\eta$ is the damping coefficient of the molecule. When one tries to represent temperature as a function of laser intensity and also as a function of wavelength, one finds that $T\left(I_{\text {laser }}, \lambda_{\text {laser }}\right)=\hbar \omega / Q^{\prime} k_{B}$ where $Q^{\prime}=\ln [1+$ $\left.1 /\left(n-I_{\text {laser }} / S\right)\right]$. Calculation of temperature from this equation shows that it increases linearly with laser intensity and decreases exponentially with laser wavelength. The phenomenon of the linear increase of the molecule temperature with laser intensity is expected to work only for low level excitation as proposed by Bowden et al. However, this model fails at high laser power where the relationship clearly shows non-linearity.

An energy-balance equation was presented by Haggerty and Cannon [11] in 1981. This equation states that the energy absorbed by the aerosol from the laser beam is equal to the energy used to raise its temperature, the energy used to induce chemical reactions and other heat transfer losses such as radiation, convection among others. This equation is given here as

$$
\begin{aligned}
I_{0} & \Delta A e^{-\sum \alpha_{i} p_{i} x}\left[1-e^{-\sum \alpha_{i} p_{i} \Delta x}\right] \\
& =C_{p} \frac{\aleph}{V} \Delta V \frac{d T}{d t}+\Delta H \Delta V \frac{d \aleph}{d t}+\text { heat_transfer_losses }
\end{aligned}
$$

$I_{0}$ is the input laser intensity in $\mathrm{W} / \mathrm{cm}^{2}, \Delta V$ is the volume element of the precursor aerosol, $\Delta A$ is the cross-sectional area of the aerosol as seen by the laser beam, $\alpha_{i}$ is the absorption coefficient of the $i$ th species, $p_{i}$ is the partial pressure of the $i$ th species, $x$ is the distance of the aerosol volume element from the window, $\Delta x$ is the thickness of the volume element, $C_{p}$ is the heat capacity of the gas, $\aleph / V$ is the molar density in the volume element, $d T / d t$ is the rate of change of temperature, $\Delta H$ is the heat of reaction in joule/mole, and $d \aleph / d t$ is the moles of gas reacting per unit time per unit volume. The schematic illustration in Fig. 1 
Fig. 1 A schematic illustration of the laser-beam-aerosol interaction zone shows precursor droplets erupting into a flame and decomposing into nano-particles. The volume and surface area of the flame are calculated from an elemental volume $\Delta V=\Delta A \Delta x$ and elemental surface $\int_{\text {surface }} d A$, respectively. In the insets (a) and (b) are the proposed illustrations of how the laser photons selectively interact with $\mathrm{O}-\mathrm{C}$ electron gas (bonds)

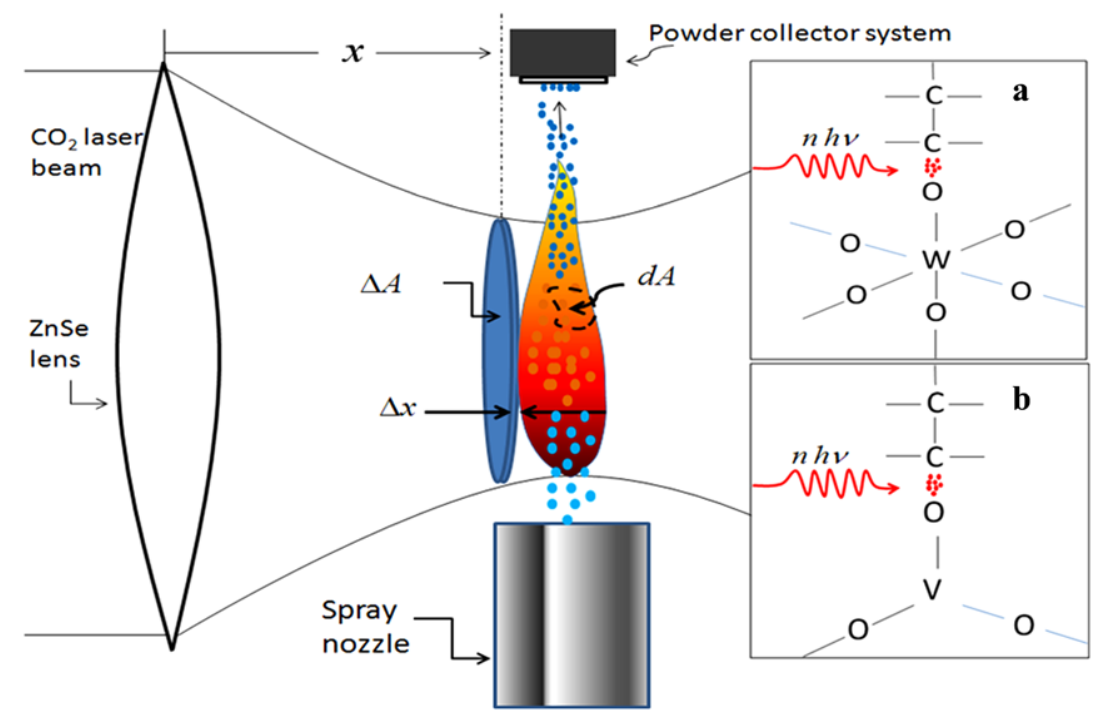

depicts the volume and surface calculation on the flames observed.

This energy balance equation has been difficult to solve. There are no known relations for the number, $\aleph$, of molecular species present in the beam at a particular period of time and let alone how many of them change from one chemical species, $d \aleph / d t$, to the other. Haggerty and Cannon themselves did not attempt to solve this equation let alone to determine the absolute temperatures in their flames.

On the other hand, experimental data on laser power and temperatures of benzene was presented by Tenegal et al. [12] in 2001 but they could not explain their findings by any physical model.

The theoretical attempt by El-Diasty et al. [13] to describe the laser pyrolysis of silane is worth noting. However, the role of the laser intensity and wavelength on the flame temperature was not pursued by El-Diasty et al.

Recently, another attempt to measure and model the laser pyrolysis of iron nano-particles via the aerosol theory was made by Bomati-Miguel et al. [14]. Their laser pyrolysis modeling involved the particle size of the powdered products as a function of laser intensity but not as a function of the temperature of these products while in the laser beam. They have admitted that measuring temperature is difficult in the laser pyrolysis process because the presence of the laser precludes the introduction of any device in the reaction zone. Only optical systems were seen to be useful, but their effectiveness is restricted to strong light-emission processes obtained at high laser intensities [15, 16]. Their laser pyrolysis process took place under low-emission conditions, which made the standard infrared pyrometers useless. In their work, they claim they solved this problem by measuring the temperature profile in the gas mixture along the reaction plume immediately after irradiation by means of a series of five equidistant K-type thermocouples. From the linear plot of the reciprocal temperature $1 / T$ versus the distance from the nozzle, the maximum temperature increase $T_{\max }$ was obtained as the intercept at the origin. This approach has limitations: (1) use of thermocouples has already been mentioned to be dubious (2) the fast clogging of the thermocouples by the iron powders influence the temperature very much. Bomati-Miguel et al. could not determine the exact temperatures at any specific point in the flame. Moreover, the discussion of laser wavelength is ignored in Bomati-Miguel et al. work as well as in many studies on laser pyrolysis so far. The connection between laser intensity and laser wavelength on the temperature of the starting aerosol and hence the particle size of the end product distinguishes the present discussion from many previous studies.

\section{Theoretical considerations}

Albeit the difficulty, the Haggerty-Cannon energy balance equation can be used to describe the temperature changes and their temporal evolutions thereof. For this reason, we have simplified (1) by noting that the first two terms on the right are only important for the transient part of the temporal temperature. The transient part of the temporal temperature can be worked out from (1) by noting that the term $\Delta H \Delta V \partial \aleph / \partial t$ does not contribute to the change in temperature since this is the latent heat term. The heat transfer loss terms are radiation and convection rate only.

\subsection{Heat loss by convection and radiation}

The power lost per unit time by the droplet through radiation in vacuum is $\varepsilon \sigma A T^{4}$ where $\varepsilon$ is the emissivity of the constituents in the flame, $\sigma$ is the Stefan-Boltzmann's constant, and $A$ is the surface area of the flame. 
Determining the surface area of any flame is admitted very difficult. Surface areas of flame cones were calculated from motion-picture frames, with the shadow-gram and emission oscillogram registered simultaneously on the same film by Afanas'ev et al. [17]. Most estimates in literature regard the flame shape as a cone [18]; herein we regard our flames as being close to two cones joined at their bases as illustrated in the inset of Fig. 1 where the total surface area $A$ is given as $A=\left(\pi\left(L_{1}+L_{2}\right)(d / 2)=\right.$ $\pi\left(\left[(d / 2)^{2}+h_{1}^{2}\right]^{1 / 2}+\left[(d / 2)^{2}+h_{2}^{2}\right]^{1 / 2}\right)(d / 2)$ where $d$ is the diameter of the flame at the waist, $L$ is the length of the flame cone from apex to the waist and $h$ is the height of the flame cone from apex to the waist. The total surface area of such a shape can gives a good estimate of the surface area. The actual surface area fluctuates in time. In such a case, the root of the mean of the square (rms) surface area must be determined. In the present case, the flame is stable, and hence its surface area is almost constant with time.

Also, since the flame is not in vacuum, but rather is surrounded by $\mathrm{C}_{2} \mathrm{H}_{2}$ and $\mathrm{Ar}$ gases, the radiation loss rate is modified [19] to $(\partial C / \partial t)_{\text {radiation }}=\varepsilon \sigma A\left(T^{4}-T_{s}^{4}\right)$ where $T_{S}$ is the temperature of the surrounding carrier gases. The emissivity $\varepsilon$ is calculated from experimental transmittance, and hence absorption spectra in the spectral range as defined in the following equation:

$$
\begin{gathered}
\varepsilon=\frac{\int_{\omega_{1}}^{\omega_{2}} \alpha(\omega) L_{\mathrm{bb}}(\omega) d \omega}{\left(\int_{\omega_{1}}^{\omega_{2}} L_{\mathrm{bb}}(\omega) d \omega\right)} \\
\omega_{1}=909 \mathrm{~cm}^{-1} ; \quad \omega_{2}=1250 \mathrm{~cm}^{-1}
\end{gathered}
$$

Here, $\alpha(\omega)$ is the absorbance of the liquid at a frequency in wave numbers $\omega\left(\mathrm{cm}^{-1}\right)$ and $L_{\mathrm{bb}}(\omega)$ represents the blackbody radiation spectrum in terms of wave numbers. The integration limits $\omega_{1}$ and $\omega_{2}$ are the FTIR detector ranges of $909 \mathrm{~cm}^{-1}$ (a wavelength of $12 \mu \mathrm{m}$ ) and $1250 \mathrm{~cm}^{-1}$ (a wavelength of $7.5 \mu \mathrm{m})$, respectively.

The rate of energy loss through convection is given [19] by the very well-known relation $(\partial C / \partial t)_{\text {convection }}=$ $\kappa_{\text {conv }} A\left(T-T_{S}\right)$ where $\kappa_{\text {conv }}$ is the coefficient of convection. Since there is no contact with the wall of the chamber, the energy loss to conduction, $\kappa_{\text {cond }} A(\partial T / \partial r)$, is zero.

\subsection{Transient temperature as a function of laser intensity}

We write (1) by simplifying the left-hand side to the fraction $\mu P_{\text {laser }}$ of the laser power absorbed by the spray molecule. We also replace the heat losses on the right-hand side with heat losses by radiation and convection only. This equation then changes to

$$
\begin{aligned}
\mu P_{\text {laser }}= & C_{p} \frac{\aleph}{V} \Delta V \frac{d T}{d t}+\Delta H \Delta V \frac{d \aleph}{d t} \\
& +\varepsilon \sigma A\left(T^{4}-T_{s}^{4}\right)+\kappa_{\mathrm{conv}}\left(T-T_{s}\right)
\end{aligned}
$$

For a photochemical process, the change of chemical species $d \aleph / d t$ from one species to the other happens without such rapid change in temperature. Ideally, a photochemical process happens without any change in temperature. Note that in the real case, $d T / d t \neq 0$, thus there still exists a small change in temperature $T_{\tau}$ in a typical photochemical process. We then can rewrite (3) for the photochemical process to a differential equation:

$\beta_{0} \frac{d \aleph}{d t}+\beta_{1} \aleph+\beta_{2}=0$

$\beta_{0}=\Delta V \Delta H ; \quad \beta_{1}=\frac{C_{p} \Delta V}{V} T_{\tau}$

$\beta_{2}=A \varepsilon \sigma T_{\tau}^{4}-\mu P_{\text {laser }}-A \varepsilon \sigma T_{s}^{4}-\kappa_{\mathrm{conv}} T_{s}$

Given the fact that the number of molecules $\aleph$ converting from precursor to product is zero at $t=0$ when the laser power is also zero, (4) will have a solution given as

$$
\begin{aligned}
\aleph(t)= & \frac{\mu P_{\text {laser }}+A \varepsilon \sigma T_{s}^{4}+\kappa_{\mathrm{conv}} T_{s}-A \varepsilon \sigma T_{\tau}^{4}}{C_{p} \Delta V} \\
& \times V\left(1-e^{-\frac{C_{p} \Delta V}{V \Delta H} t}\right)
\end{aligned}
$$

For a photothermal process we can analyze (3) as follows: if the number of molecules passing through the pyrolysis chamber per unit time is kept constant and the rates of reaction are stable at some rate $d \aleph / d t=\aleph_{\tau}$, we can simplify (3) to the following differential equation:

$\alpha_{0} \frac{d T}{d t}+\alpha_{1} T^{4}+\alpha_{2} T+\alpha_{3}=0$

$\alpha_{0}=\frac{C_{p} \aleph \Delta V}{V} ; \quad \alpha_{1}=A \varepsilon \sigma ; \quad \alpha_{2}=\kappa_{\mathrm{conv}}$

$\alpha_{3}=\Delta V \Delta H \aleph_{\tau}-\mu P_{\text {laser }}-A \varepsilon \sigma T_{s}^{4}-\kappa_{\mathrm{conv}} T_{s}$

Finding the solution of (6) in $T$ gives us $T$ as a func-

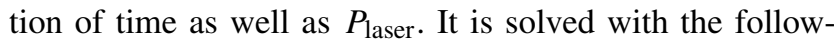
ing boundary conditions: at $t=0, T=T_{s}$, and at $t \rightarrow \infty$, $T \rightarrow T_{\text {eq }}$. We try an expression for temperature as function of time such as $T(t)=T_{S}+\left(T_{\max }-T_{S}\right) \exp [-\rho t]$. When this is substituted in (5), we find a polynomial in $\rho$ to the power of 4 . This is circumvented by noting that the transient temperature takes place at very short time $t$ for which case the $\exp [-4 \rho t]$ term can be estimated to the first linear term after the Taylor expansion of this exponential function. We therefore find $T$ as function of $t$ as follows:

$$
\begin{aligned}
& T(t)=T_{s}+\left(T_{\mathrm{eq}}-T_{S}\right)(1 \\
& -\exp \left[-P \log \left(\frac{\exp [A t V \varepsilon \sigma]}{V} \frac{+\mu P_{\text {laser }}-\Delta V \Delta H \aleph_{\tau}+A \varepsilon \sigma T_{s}^{4}+\kappa_{\mathrm{conv}} T_{s}}{\aleph \Delta V C_{p}} t\right)\right. \\
& \left.\left.\quad+\frac{A V \varepsilon \sigma . t}{\aleph \Delta V C_{p}}\right]\right)
\end{aligned}
$$


The operation, $P \log [z]$ in (7), shortened for Product $\log [z]$, is a Mathematica command which gives the solution of $w$ in the expression $z=w e^{w}$.

The final "equilibrium" temperature of molecules that are entrained into a $\mathrm{CO}_{2}$ laser beam found either by letting $t \rightarrow \infty$ as can be seen in (6). In a typical experiment, the equilibrium is reached in a fraction of a second after the laser beam is switched-on as will be shown in the experimental sections that follow. During the cooling cycle, temperature goes to surrounding temperature if one sets $P_{\text {laser }}=0$ in (2). For the known values of the Stefan-Boltzmann's constant, $\sigma$, volume of the interaction zone, $V=A . \Delta V$, precursor flow rate, $\aleph_{\tau}$, surrounding temperature, $T_{s}$, and from the fittings of heating and cooling equations above to the experimental data, the following parameters are determined: specific heat capacity $C_{p}$ of the starting material, the enthalpy of the reaction, $\Delta H$, the convection co-efficient, $\kappa_{\text {conv }}$, of the surrounding, and the fraction of the laser beam absorbed by the precursor liquid, $\mu$.

\subsection{Temperature of the flame as a function of laser wavelength and laser power}

The analysis in Sect. 3.2 assumed largely that the laser excitation was equivalent to any invariant heat source. No oscillations in the laser field were considered. However, interesting phenomena such as selective dissociation, and hence new materials show up when the laser beams of varying wavelengths are used. Therefore, an attempt to understand this aspect of laser-material interaction is important. After determining parameters, $C_{p}, \Delta H, \kappa_{\mathrm{conv}}, \mu$, from the previous discussion, new parameters such as saturation power $P_{\text {sat }}$, resonant wavelength, $\lambda_{\text {res }}$, and damping coefficients, $\eta$, of the near-to-resonance $\mathrm{O}-\mathrm{C}$ bonds in the precursor materials can be easily determined.

We start this analysis by realizing that the net temperature at any time, $t$, at a particular point in the flame, can be calculated from the sum of heating rates and cooling rate. There are two main heating rates $\partial H_{1} / \partial t$ and $\partial H_{2} / \partial t$ being opposed by two cooling rates $\partial C_{1} / \partial t$ and $\partial C_{2} / \partial t$. The heating rate, $\partial H_{1} / \partial t$, is as a result of the excitation by the laser. The Haggerty-Cannon equation (1) gives this heating rate only in terms of the laser intensity without the laser wavelength. We rederive this heating rate to incorporate a varying laser field. We do this by calculating the force that the photon exerts on the electron, the collective force that the electrons of an atom exert on that atom as a consequence of the photon excitation and the consequent forces between atoms which cause bond dissociation of the molecules. $\partial H_{2} / \partial t$ is the heating rate due to photon recoil. Excited electrons have to come to ground state by spontaneous or stimulated emission of photons. In the process, the electrons recoil with some finite velocity. The collective force, due to electrons' photon recoil, impacts on atoms and their bonds in the molecules in the similar manner as the $\partial H_{1} / \partial t$ mechanism only with a less magnitude.

For the molecules to stay in thermal equilibrium and for there to exist a measurable constant temperature, there must be an equal net cooling rate, $\partial C_{1} / \partial t+\partial C_{2} / \partial t$. These cooling rates are the radiation and convection heat loss rates given in the previous sections.

3.4 Heating rate due to droplet absorption of laser photons, $\partial H_{1} / \partial t$

The two starting liquids are dissolutions of $\mathrm{VCl}_{3}$ (green) and $\mathrm{WCl}_{6}$ (dark-blue) powders in ethanol to form green $\mathrm{VCl}^{3-x}\left(\mathrm{OC}_{2} \mathrm{H}_{5}\right)_{x}$ and light blue $\mathrm{WCl}^{6-x}\left(\mathrm{OC}_{2} \mathrm{H}_{5}\right)_{x}$, respectively, $[19,20]$. The $\mathrm{V}-\left(\mathrm{OC}_{2} \mathrm{H}_{5}\right)_{3}$ and $\mathrm{W}-\left(\mathrm{OC}_{2} \mathrm{H}_{5}\right)_{6}$ form after a few weeks when left at room temperature. When the $\mathrm{CO}_{2}$ laser photons of frequency $\omega$ emits light they exert forces on a group of electrons as illustrated in Figs. 1(a) and 1(b). The electrons mediate between the atoms. The atoms, in the present starting materials, have at least one resonant bond, or a close-to-resonance electron gas identified by infrared spectroscopy to be between atoms $\mathrm{O}$ and $\mathrm{C}$ in each case. The reduced masses loaded on each bond in these molecules are given as follows:

$$
\begin{aligned}
\left(\frac{1}{\mu^{*}}\right)_{\mathrm{V}_{\left(\mathrm{OC}_{2} \mathrm{H}_{5}\right)_{3}}=} & \frac{1}{m_{\mathrm{V}, \mathrm{O}}}+\frac{1}{m_{\mathrm{C}, \mathrm{H}}} \\
= & \frac{1}{\sum m_{\mathrm{V}}+3 \sum m_{\mathrm{O}}} \\
& +\frac{1}{6 \sum m_{\mathrm{C}}+15 \sum m_{\mathrm{H}}}
\end{aligned}
$$

$$
\begin{aligned}
\left(\frac{1}{\mu^{*}}\right)_{\mathrm{W}\left(\mathrm{OC}_{2} \mathrm{H}_{5}\right)_{6}}= & \frac{1}{\sum m_{\mathrm{W}}+6 \sum m_{\mathrm{O}}} \\
& +\frac{1}{12 \sum m_{\mathrm{C}}+30 \sum m_{\mathrm{H}}}
\end{aligned}
$$

By employing (7) and (8) in calculating the effective masses loading the $\mathrm{O}-\mathrm{C}$ bonds in our starting materials$\mathrm{V}-\left(\mathrm{OC}_{2} \mathrm{H}_{5}\right)_{3}$ and $\mathrm{W}-\left(\mathrm{OC}_{2} \mathrm{H}_{5}\right)_{6}$-one obtains $\left(\mu_{\mathrm{O}-\mathrm{C}}^{*}\right)_{\mathrm{V}}=$ 24.47849 a.m.u. and $\left(\mu_{\mathrm{O}-\mathrm{C}}^{*}\right)_{\mathrm{W}}=27.14701$ a.m.u, respectively. Tenegal et al. (2001) considered the benzene molecule and its temperature-laser power data have been included in this discussion. Similarly, the effective mass for the benzene molecule is $\left(\mu_{\mathrm{C}=\mathrm{C}}^{*}\right)_{\text {benzene }}=16.5274$ a.m.u.

If the specific electron gas has a damping coefficient $\eta$ and an effective elastic constant given by $\mu \omega_{0}^{2}$, the onedimensional forced damped harmonic oscillator equation of motion for the displacement of this electron cloud and hence the $\mathrm{O}$ and $\mathrm{C}$ atoms can written thus

$\frac{d^{2} r}{d t^{2}}+\eta \frac{d r}{d t}+\omega_{0}^{2} r=\frac{q}{\mu} E_{0} e^{i \omega t}$ 
Here, $q$ is the total charge on the electron gas and $E_{0}$ is the laser photon's maximum electric field. We substitute the trial function $r=E_{0} e^{i(\omega t-\phi)}$ and expand (9) in order to equate real and imaginary parts to obtain $\tan \phi=$ $\eta \omega /\left(\omega_{\text {res }}^{2}-\omega^{2}\right)$ and the amplitude $r_{0}=q E_{0} / 2 \mu \omega\left[\left(\omega_{\text {res }}^{2}-\right.\right.$ $\left.\left.\omega^{2}\right)^{2}+\gamma^{2} \omega^{2}\right]$. In the limit as the excitation frequency gets near resonance with a detuning, $\Delta=\omega-\omega_{\text {res }}$, the following expressions for phase angle $\phi$, and vibrational amplitude $x_{0}$ apply: $\tan \phi=\eta \omega / 2 \Delta$ and $q E_{0} / 2 \mu \omega_{\text {res }}\left[\Delta^{2}+\right.$ $\left.\gamma^{2} / 4\right]^{1 / 2}$. The average power $P_{\mathrm{av}}$ absorbed by the vibrating electron can be derived from $e E d x / d t$ whose imaginary part amounts to zero whereas the real part yields: $P_{\mathrm{av}}=e E \omega r_{0} \cos \phi\left[(1 / T) \int_{T} \sin 2 \omega t d t\right]$. When the expression for $r_{0}$ and right trigonometric identities are employed, the average power absorbed by the molecule can be shown to simplify to:

$P_{\mathrm{av}}=\frac{e^{2}}{\mu^{*}} \frac{\eta}{\left(4 \Delta^{2}+\eta^{2}\right)} E_{0}^{2}$

This average absorbed power in conjunction with the standard expressions for intensity, $I=\varepsilon_{0} c E_{0}^{2}$ and saturation intensity $I_{\text {sat }}=\varepsilon_{0} \mu c^{2} \eta^{3} h / e^{2} \lambda=1.23 \times 10^{19} \mu^{*} \eta^{3}$ can be used to derive the heating rate due to absorption in terms of $\left(I / I_{\text {sat }}\right)$. Since $\left(I / I_{\text {sat }}\right)$ is equal to $\left(P_{\text {laser }} / P_{\text {laser }}^{\text {sat }}\right)$, the heating rate due to laser photon absorption by the aerosol spray can be given as

$$
\left(\frac{\partial H}{\partial t}\right)_{\text {absorption }}=\frac{2 e^{4} h \eta^{3} \omega}{\mu^{* 2}\left(\eta^{2}+4 \Delta^{2}\right)}\left(\frac{P_{\text {laser }}}{P_{\text {laser }}^{\text {sat }}}\right)
$$

For the precursors under study, the relationships between saturation intensity $I_{\text {sat }}$ and the damping coefficient $\eta$ can be estimated by substituting the effective mass on the sensitive bonds above thus: $\left(I_{\text {sat }}\right)_{\mathrm{V}}=1.23 \times 10^{19} \times 24.47 \times$ $1.67 \times 10^{-27} \eta^{3}=5.029 \times 10^{-7} \eta^{3}$ for vanadium ethoxide; $\left(I_{\text {sat }}\right)_{\mathrm{W}}=1.23 \times 10^{19} \times 27.147 \times 1.67 \times 10^{-27} \eta^{3}=$ $5.579 \times 10^{-7} \eta^{3}$ for tungsten ethoxide and $\left(I_{\text {sat }}\right)_{\text {benzene }}=$ $1.23 \times 10^{19} \times 24.47 \times 1.67 \times 10^{-27} \eta^{3}=3.397 \times 10^{-7} \eta^{3}$ for benzene. These estimations are valid for a $10.6 \mu \mathrm{m} \mathrm{CO} 2$ laser excitation only.

Damping constants for many atomic systems have been determined in the past when $\mathrm{HeNe}$ and Ar lasers are employed. For $\mathrm{Na}$ atoms the value of $\eta=6.17 \times 10^{7} \mathrm{~s}^{-1}$ for damping constant was reported [21] so that if our starting materials were excited by the $\mathrm{HeNe}$ and Ar lasers, their saturation intensities would be of the order of $\sim 30 \times$ $10^{14} \mathrm{~W} / \mathrm{m}^{2}, 33 \times 10^{14} \mathrm{~W} / \mathrm{m}^{2}$ and $19 \times 10^{14} \mathrm{~W} / \mathrm{m}^{2}$, respectively. More accurate than these saturation intensity values will be given in the results section of this work.

\subsection{Heating rate by photon recoil, $\partial H_{2} / \partial t$}

Another source of heat in the atoms is as a result of their photon emission after excitation. Excited atoms return to ground state by spontaneous emission or stimulated emission with the two emissions having equal probability; both absorption and emission are random. Hence, their average momenta are zero but the square of the momenta $h k$ is finite given [22] in terms of the $N$ quantized steps as

$$
\begin{aligned}
& \left(\frac{\partial H}{\partial t}\right)_{\text {recoil }} \\
& \quad=\frac{1}{2 \mu^{*}} \frac{d \overline{p^{2}}}{d t}=\frac{(\hbar k)^{2}}{2 \mu} \frac{\eta}{1+(2 \Delta / \eta)^{2}}\left(\frac{P_{\text {laser }}}{P_{\text {laser }}^{\text {sat }}}\right)
\end{aligned}
$$

3.6 Thermal equilibrium in the flame

The steady state part of the temporal temperature can be worked out by balancing the heating rates and the cooling rates. When the flame reaches thermal equilibrium, the absorption rate in (12) plus the photon recoil heating rate in (13) is balanced by the power lost through radiation and convection. Since there is no contact between the flame and the wall of the chamber, the energy loss to conduction is zero. Hence, the energy balance equation at steady state or when thermal equilibrium is attained can be given thus

$$
\begin{gathered}
\frac{2 e^{4} h \eta^{3} \omega}{\mu^{* 2}\left(\eta^{2}+4 \Delta^{2}\right)}\left(\frac{P_{\text {laser }}}{P_{\text {laser }}^{\text {sat }}}\right)+\frac{\left(\hbar k_{B}\right)^{2}}{2 \mu^{*}} \frac{\eta}{1+(2 \Delta / \eta)^{2}}\left(\frac{P_{\text {laser }}}{P_{\text {laser }}^{\text {sat }}}\right) \\
-\varepsilon \sigma A\left(T^{4}-T_{s}^{4}\right)-\kappa_{\text {conv }}\left(T-T_{S}\right)=0
\end{gathered}
$$

The polynomial in $T$ in this equation is solvable, especially, for the case where we neglect convection. In such a case, temperature, $T$ has the solution:

$$
\begin{aligned}
T\left(P_{\text {laser }}, \lambda\right)= & \left(T_{s}^{4}+\frac{1}{\varepsilon \sigma A}\left(\frac{2 e^{4} \hbar \eta^{3}(c / \lambda)}{\mu^{* 2}\left(\eta^{2}+4\left(\frac{c}{\lambda_{\text {res }}}-\frac{c}{\lambda}\right)^{2}\right)}\right.\right. \\
& \left.\left.+\frac{\left(\hbar k_{B}\right)^{2}}{2 \mu^{*}} \frac{\eta}{1+\left(\frac{2}{\eta}\left(\frac{c}{\lambda_{\text {res }}}-\frac{c}{\lambda}\right)\right)^{2}}\right)\left(\frac{P_{\text {laser }}}{P_{\text {laser }}^{\text {sat }}}\right)\right)^{1 / 4}
\end{aligned}
$$

Neglecting convection simplifies the solution of (14) and also gives a rough idea about the magnitude of some unknown parameters such as $P_{\text {sat }}, \eta$ for the precursor liquids. However, fitting (14) to the experimental data is not expected to yield a perfect fit. This is due to the fact that in the typical laser pyrolysis, the laser heating does not occur in vacuum where cooling rate by radiation dominates over convection rate. The pyrolysis takes place in an ambience with $\mathrm{C}_{2} \mathrm{H}_{2}, \mathrm{Ar}$, and $\mathrm{O}_{2}$ gases flowing at specified flow rates. Therefore, cooling rate by convection is supposed to be equally important. In this case then, one can obtain an improved expression for temperature of the flame $T$ at certain 
Fig. 2 (a) The IR camera and IR spectrometer data acquistion setup around the laser pyrolysis experimental site.

(b) Photograph of the laser pyrolysis chamber taken from a normal UV-Visibleregion camera. No flame is seen by this camera. (c) A typical image of the once-invisible flame taken from the IR camera. A flame is now visible. (d) A schematic representation of how the surface area of the flame is calculated by assuming two cones joined at their bases

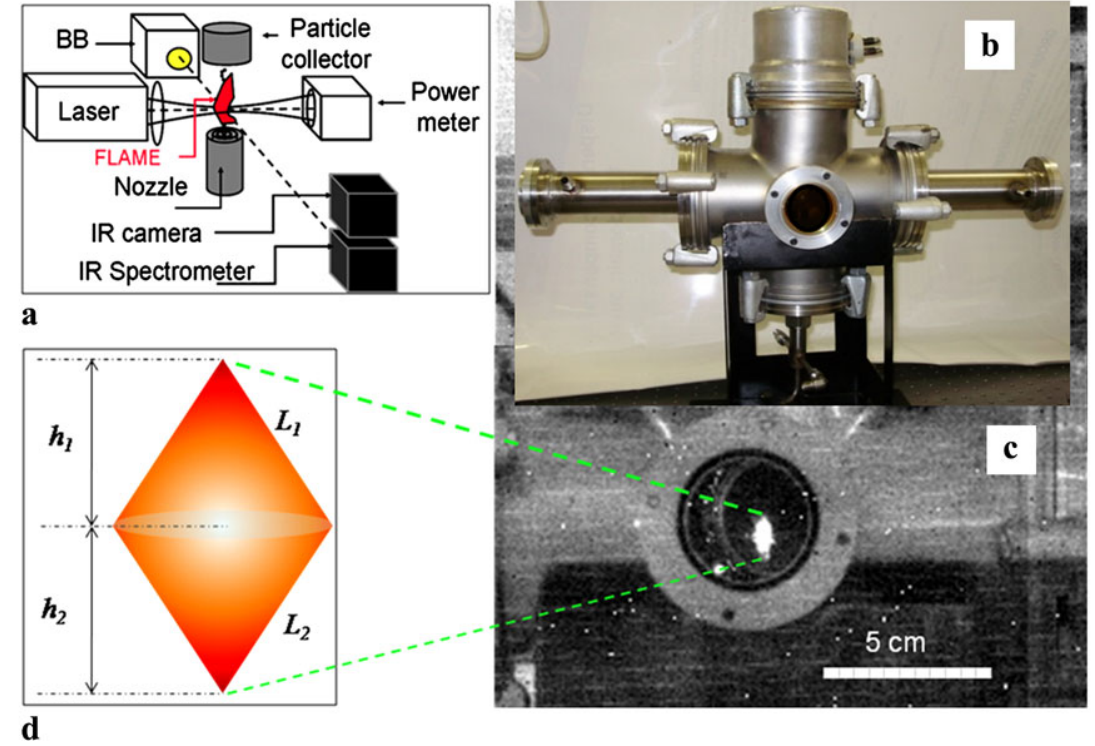

point as a function of laser intensity $P_{\text {laser }}$ and laser wavelength, $\lambda$ as:

$$
\begin{aligned}
T( & \left.P_{\text {laser }}, \lambda\right) \\
= & \frac{1}{2}\left[\sqrt{\frac{B}{2^{1 / 3} 3^{2 / 3}}-\frac{4\left(\frac{2}{3}\right)^{1 / 3} f}{\varepsilon \sigma A_{\text {surf }} B}}\right. \\
& -\sqrt{\frac{4\left(\frac{2}{3}\right)^{1 / 3} f}{\varepsilon \sigma A_{\text {surf }} B}-\frac{B}{2^{1 / 3} 3^{2 / 3}}+\frac{\varepsilon \sigma A_{\text {surf }} \sqrt{\frac{B}{2^{1 / 3} 3^{2 / 3}}-\frac{4\left(\frac{2}{3}\right)^{1 / 3} f}{\varepsilon \sigma A_{\text {surf }} B}}}{\left(\varepsilon \kappa_{\text {conv }}\right.}} \\
B= & \left.\frac{9 \kappa_{\text {conv }}^{2}}{\left(\varepsilon \sigma A_{\text {surf }}\right)^{2}}-\frac{\sqrt{3} \sqrt{27 \kappa_{\text {conv }}^{2}+256 \varepsilon \sigma A_{\text {surf }} f^{3}}}{\left(\varepsilon \sigma A_{\text {surf }}\right)^{3}}\right)^{1 / 3} \\
f= & \frac{2 e^{4} \hbar \eta^{3} c}{\mu^{* 2} \lambda\left(\eta^{2}+4\left(\frac{c}{\lambda_{\text {res }}}-\frac{c}{\lambda}\right)^{2}\right)}\left(\frac{P_{\text {laser }}}{P_{\text {laser }}^{\text {sat }}}\right) \\
& +\frac{\left(\hbar k_{B}\right)^{2}}{2 \mu^{*}} \frac{\eta}{1+\left(\frac{2\left(\frac{c}{\lambda_{\text {res }}}-\frac{c}{\lambda}\right)}{\eta}\right)^{2}}\left(\frac{P_{\text {laser }}}{P_{\text {laser }}^{\text {sat }}}\right) \\
& -\varepsilon \sigma A T_{s}^{4}-\kappa_{\text {conv }} T_{s}
\end{aligned}
$$

Three unknown parameters $-P_{\text {sat }}, \lambda_{\text {res }}$, and $\eta-$ need to be determined from this equation; the rest of the parameters are known from the temporal temperature data in (7).

\section{Methodology}

4.1 Infrared spectra of the invisible $\mathrm{VCl}^{3-x}\left(\mathrm{OC}_{2} \mathrm{H}_{5}\right)_{x}$ and $\mathrm{WCl}^{6-x}\left(\mathrm{OC}_{2} \mathrm{H}_{5}\right)_{x}$ flames

A CEDIP Jade infrared camera sensitive from 7.5 to $12 \mu \mathrm{m}$ wavelength range was employed in imaging the onceinvisible flame at the interaction zone between the laser beam and the aerosol molecules as shown in the experimental schematic set-up in Fig. 1(a). The $\mathrm{VO}_{2}$ and $\mathrm{WO}_{3}$ precursor liquids were prepared from $\mathrm{VCl}_{3}$ and $\mathrm{WCl}_{6}$ powders in ethanol to produce respectively $\mathrm{V}-\left(\mathrm{OC}_{2} \mathrm{H}_{5}\right)_{3}$ and $\mathrm{W}-$ $\left(\mathrm{OC}_{2} \mathrm{H}_{5}\right)_{6}$ solutions [23-25]. These liquids were sprayed into the laser beam and their powders were collected on a glass substrate. The flame at the laser-beam-aerosol interaction zone could be clearly seen as shown in the photograph in Figs. 2(b) and 2(c). The IR camera also allowed for the capture of movies of these flames so that temperature-time data could be attained this way. Also, IR spectra of such flames compared with the blackbody spectrum were obtained (Fig. 3). The signal from the flame consists of scattering by the aerosol molecules and particles of the $\mathrm{CO}_{2}$ laser beam [amplified from region A of Fig. 3 into inset (ii)] and the emission from the particles themselves. The two schematic illustrations in inset (i) illustrates that the signal the IR spectrometer sees when the laser is OFF is simply $\tau_{\mathrm{BB}}(\omega)$ whereas, when the laser is $\mathrm{ON}$, it is $\tau^{\prime}=\varepsilon(\omega)+\tau_{\mathrm{BB}}(\omega)+\rho_{\text {laser }}(\omega)$ where $\rho_{\text {laser }}(\omega)$ is the reflection (scattering) from the laser beam.

Clearly a laser peak at $942.78 \mathrm{~cm}^{-1}(10.6 \mu \mathrm{m})$ shows up in the spectrum as amplified in Fig. 3 inset (ii). The aerosol is less transmitting (or more absorbing and hence emitting) when the laser is ON than OFF. We have amplified the main absorption regions $\mathrm{B}$ and $\mathrm{C}$ of the spectrum in Fig. 3 into Figs. 4(c) and 4(d). When the $\mathrm{CO}_{2}$ laser is OFF, one sees the aerosol hyperfine transmission peaks notably at T1024 [1024 $\mathrm{cm}^{-1}$ or $\left.9.77 \mu \mathrm{m}\right], \mathrm{T} 1096\left[1096 \mathrm{~cm}^{-1}\right.$ or $\left.9.12 \mu \mathrm{m}\right]$ in region $\mathrm{B}$ and at $\mathrm{T} 2815$ [2815 $\mathrm{cm}^{-1}$ or $\left.3.55 \mu \mathrm{m}\right], \mathrm{T} 2822$ [2822 $\mathrm{cm}^{-1}$ or $3.54 \mu \mathrm{m}$ ], T2881 [2881 $\mathrm{cm}^{-1}$ or $3.47 \mu \mathrm{m}$ ], T2895 [2895 $\mathrm{cm}^{-1}$ or $\left.3.45 \mu \mathrm{m}\right]$, and T2941 $\left[2941 \mathrm{~cm}^{-1}\right.$ or $3.40 \mu \mathrm{m}]$ in region $\mathrm{C}$. 
Fig. 3 Far-field IR spectral transmittance of the aerosol when the laser is OFF and ON showing the laser reflection peak at $A$ [amplified in insets (i) and (ii)]
Fig. 4 Absorption bands of Fig. 3 at $B$ and $C$ amplified. The amplified diagrams reveal more absorption and emission peaks

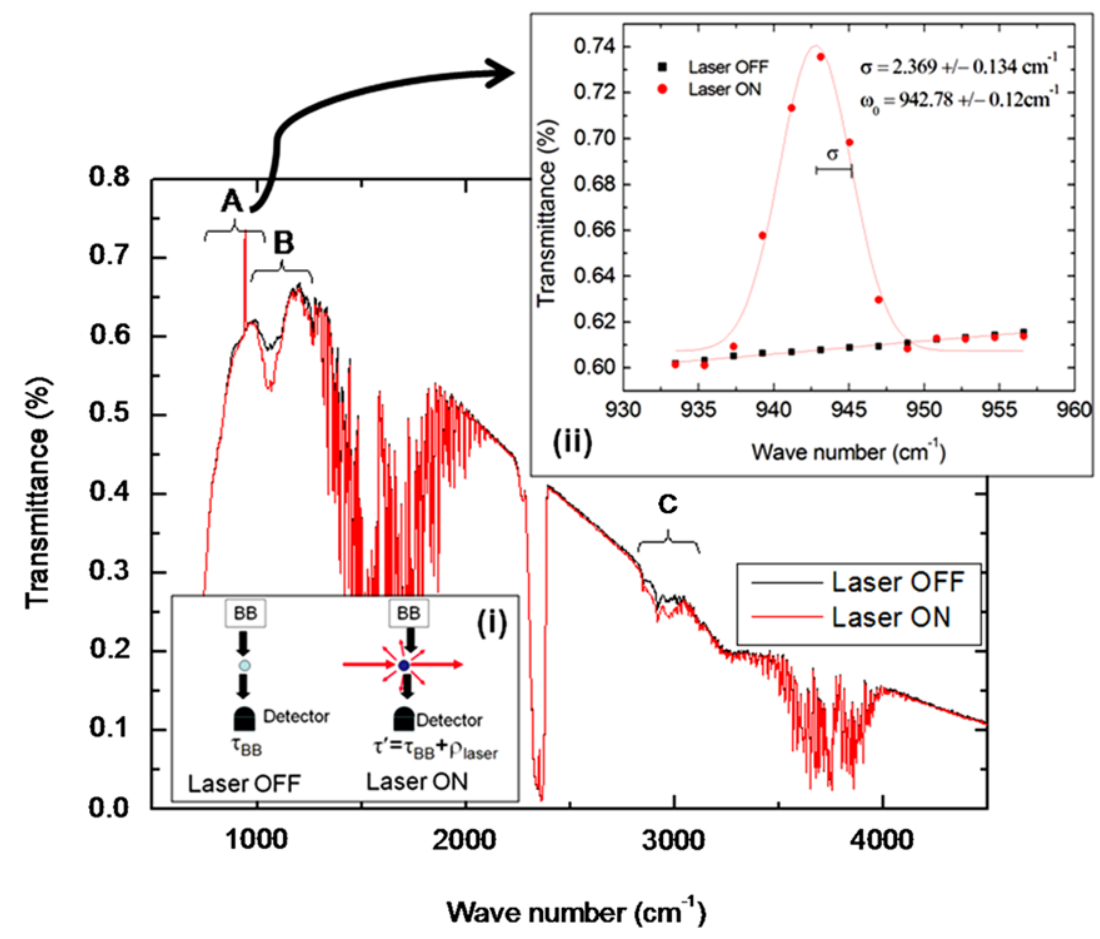

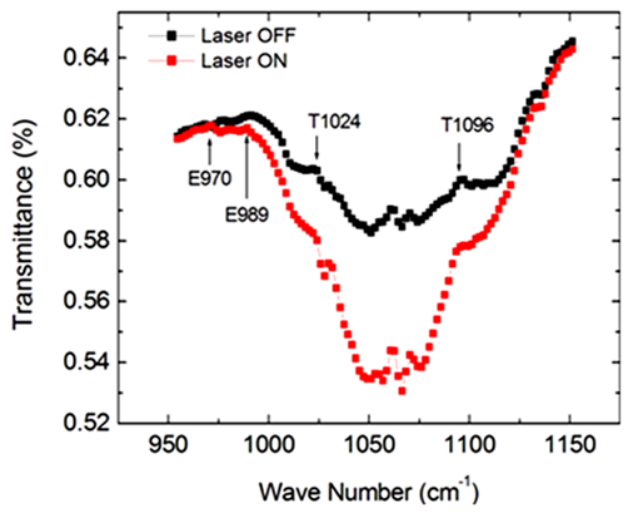

4.2 Flame emissivity determination and temperature measurements

When the laser is $\mathrm{ON}$, the absorption of the radiation from the blackbody source by the aerosol is increased in regions $\mathrm{B}$ and $\mathrm{C}$ of Fig. 4 accompanied by emission peaks notably at E970 [970 $\mathrm{cm}^{-1}$ or $\left.10.31 \mu \mathrm{m}\right]$, E989 $\left[989 \mathrm{~cm}^{-1}\right.$ or $10.11 \mu \mathrm{m}$ ], E2847 [2847 $\mathrm{cm}^{-1}$ or $3.51 \mu \mathrm{m}$ ], E2945 [2945 $\mathrm{cm}^{-1}$ or $\left.3.52 \mu \mathrm{m}\right]$, and E2983 [2983 $\mathrm{cm}^{-1}$ or $\left.3.35 \mu \mathrm{m}\right]$. Apart from hyperfine transmission and emission peaks, there are some definite absorption and emission peaks in Fig. 5(a) labeled thus: A1750 $\left(1750 \mathrm{~cm}^{-1}\right.$ or $\left.5.71 \mu \mathrm{m}\right)$ and E1625 [1625 $\mathrm{cm}^{-1}$ or $6.15 \mu \mathrm{m}$ ], respectively. Radiance from such a flame image was corrected for the laser scattering shown in the inset of Fig. 2(b) and was thus compared to the black body spectrum of known emis- sivity, $\varepsilon$ (assumed to be unity) and known temperature $900 \mathrm{~K}$.

In order to calculate "equilibrium temperature" at a particular point in the flame, either spectral emissivity of the flame is determined online using a pump probe setup in Fig. 2(a) and employing the standard Kirchoff's law of absorptivity or the effective emissivity is determined from IR spectra over a range of wavelengths of the precursor solution using a standard expression given in (2).

A second approach toward the determination of the pyrolysis flame temperature $T$ was to solve the following radiance balance equation in the direction between the IR camera and IR spectrometer on one side and the blackbody source on the other, this direction being orthogonal to the laser-power meter direction considered in (3)-(16) above and as illustrated in Fig. 2(a). This equation is a routine formula employed by the thermographers and can be written 
Fig. 5 (a) The near-field IR spectra of the $\mathrm{V}-\left(\mathrm{OC}_{2} \mathrm{H}_{5}\right)_{3}$ flame at two laser power settings $(10 \mathrm{~W}$ and $20 \mathrm{~W})$ and when the laser is OFF and ON shows an emission peak at $1625 \mathrm{~cm}^{-1}$. (b) The proposed energy diagram (on the right) summarizes the spectra and suggests multiphoton absorption for the $\mathrm{O}-\mathrm{C}, \mathrm{O}-\mathrm{H}$ and $\mathrm{C}-\mathrm{H}$ bonds to dissociate. This is followed by the major characteristic emission at $1625 \mathrm{~cm}^{-1}$. ( $N_{R}$ stands for nonradiative transitions)
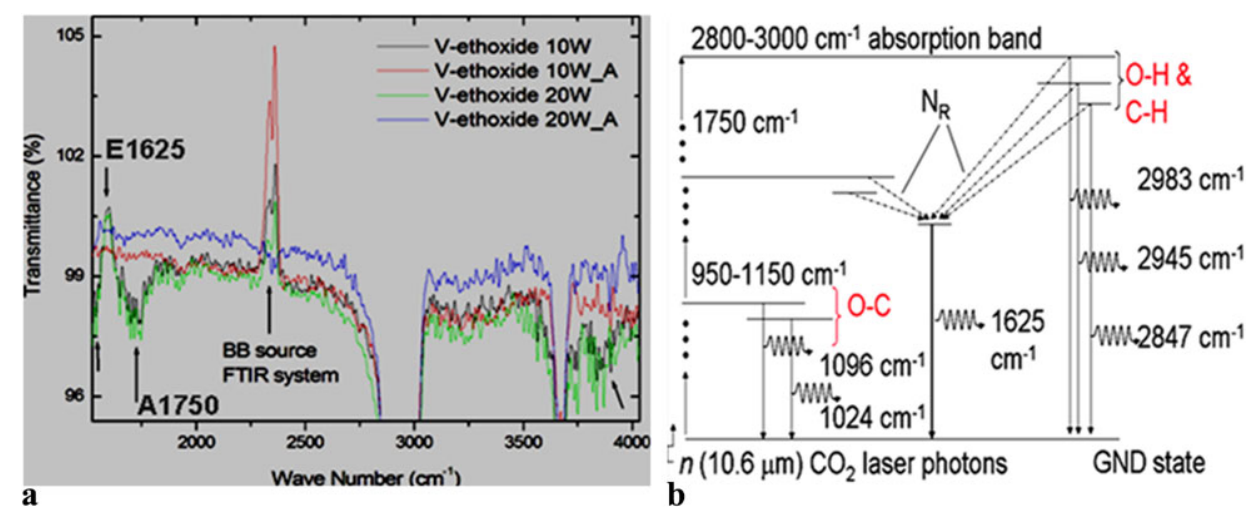

as [26]:

$$
\begin{aligned}
& \int_{7.5 \mu \mathrm{m}}^{12.0 \mu \mathrm{m}} \varepsilon_{c} L_{\mathrm{bb}}\left(T_{c}\right) S \tau_{\mathrm{ac}} d \lambda+\int_{7.5 \mu \mathrm{m}}^{12.0 \mu \mathrm{m}} \rho_{\mathrm{laser}}(\lambda) d \lambda \\
& =\int_{7.5 \mu \mathrm{m}}^{12.0 \mu \mathrm{m}} \varepsilon \cdot L_{\mathrm{bb}}(T) S \tau_{\mathrm{am}} d \lambda \\
& \quad+\int_{7.5 \mu \mathrm{m}}^{12.0 \mu \mathrm{m}}(1-\varepsilon) L_{\mathrm{bb}}\left(T_{a}\right) S \tau_{\mathrm{ae}} d \lambda+R_{\mathrm{path}}
\end{aligned}
$$

On the left of (17), we have two terms: (1) the radiance from the blackbody source and (2) the small portion of the laser beam that is scattered at right angle from its direction to the direction of the IR spectrometer and the IR camera. On the right-hand side, the three terms signify that this radiance is spent (1) in the flame (2) its ambience and (3) the path outside the laser pyrolysis chamber. Hence, the temperature $T_{c}$ is determined from the calibration curves by $T_{c}=f_{\text {cal }}(D)$, where $D$ is the measured digital level and $f_{\text {cal }}$ is the calibration curve, $\varepsilon_{c}$ is the calibration source emissivity, $L_{\mathrm{bb}}(T)$ is black body radiation of a source with temperature $T, S$ is the instrument spectral response, $\tau_{\mathrm{ac}}$ is the atmospheric transmittance during calibration, $\varepsilon$ is the measured pyrolysis flame emissivity, $T$ is the pyrolysis flame temperature, $\tau_{\mathrm{am}}$ is the spectral atmospheric transmittance between the instrument and the pyrolysis flame during measurement, $T_{a}$ is the ambient environment temperature, $\tau_{\mathrm{ae}}$ is the spectral atmospheric transmittance between the measured source and ambient environment, and $R_{\text {path }}$ is the atmospheric path radiance. The path radiance is ignored and atmospheric transmittance is assumed to be unity, since the distance between the instrument and the pyrolysis flame is small, $\sim 1.7 \mathrm{~m}$. Since the spectral response of the instrument is provided and the emissivity of the pyrolysis flame was determined from the FTIR spectrometer, the temperature of the pyrolysis flame is easily calculated from (17). The spectral emissivity used to determine the temperature of the pyrolysis flame corresponds to the spectral response of the instruments (CEDIP Jade infrared camera). The description of the method for solving (17) for measured temperature, $T$, is beyond the scope of this paper as this is a routine approach. Note also that the integration limits in this equation are band limited to the frequency range within the sensitivity of the IR camera and within the range of emission wavelengths of present flames.

\section{Results and discussion}

5.1 Measured temporal flame temperatures at the aerosol-laser interaction zone: electron heat capacities, enthalpies of formation of $\mathrm{VO}_{2}$ and $\mathrm{WO}_{3}$, and convection coefficients

After determining the emissivity values, temperatures were calculated from the radiance in the flame movies. The radiance was corrected for the laser beam reflection. From such studies, temperature-time data were obtained for various laser power settings as illustrated for the vanadium precursor liquid in Fig. 6. The continuous lines are fittings from theory which is initially fitted in the simple form $T(t)=300+\left(T_{\text {eq }}-300\right) \exp (-\beta t)$ in accordance with (7). For varying laser power values, the theoretical fit enables one to extract characteristic values of $T_{\text {eq }}$ and $\beta$. Since $\beta$ is a function of $\mu, \Delta H \aleph_{\tau}, \varepsilon, T_{s}, \kappa_{\text {conv }}$ and $C_{p}$, the values of $T_{\text {eq }}$ and $\beta$ at varying $P_{\text {laser }}$ settings enable us to find these parameters as long as we collect enough-more than seven pairs of $\left(T_{\mathrm{eq}}, \beta\right)$-data points.

From the flame movie, we estimate flame and beam constants assuming the flame to be two cones attached at their bases [as illustrated in Figs. 2(c) and 2(d)] thus: since $L_{1} \sim$ $L_{2}=4.47 \mathrm{~mm}, h_{1} \sim h_{2}=4 \mathrm{~mm}$, the cross-section of laser beam-aerosol interaction, $\Delta A=(1.61 \pm 0.24) \times 10^{-5} \mathrm{~m}^{2}$, flame surface area $A=(2.24 \pm 0.22) \times 10^{-4} \mathrm{~m}^{2}$ and flame volume $V=(1.62 \pm 1.04) \times 10^{-8} \mathrm{~m}^{3}$. From these fittings, we obtained the parameter as outlined in Table 1 .

Compared with bulk measurement for specific heat capacity of benzene and alcohols_-acetone, methanol, ethanol 
Fig. 6 Flame temperature versus time for the $\mathrm{V}-\left(\mathrm{OC}_{2} \mathrm{H}_{5}\right)_{3}$ starting material; the time variation of temperature was obtained via movies of the IR images of these flames. The lines are fittings of (10) with the parameters obtained from this fit outlined in Table 1
Fig. 7 This is a plot of the flame temperature versus laser power for $(a) \mathrm{W}-\left(\mathrm{OC}_{2} \mathrm{H}_{5}\right)_{6}$, (b) $\mathrm{V}-\left(\mathrm{OC}_{2} \mathrm{H}_{5}\right)_{3}$, and (c) benzene. The benzene data is taken from Tenegal et al. (2001) work. The red and blue lines are fittings from (24) and (25), respectively. In the inset is the temperature versus laser wavelength for $\mathrm{W}-\left(\mathrm{OC}_{2} \mathrm{H}_{5}\right)_{6}$ (blue) and $\mathrm{V}-\left(\mathrm{OC}_{2} \mathrm{H}_{5}\right)_{3}$ (orange). The poor fit in left wing in the data could be due to Mie scattering owing to droplet size effects or could be another resonant peak at lower wavelength lower than the laser tuneability limit of $9.22 \mu \mathrm{m}$
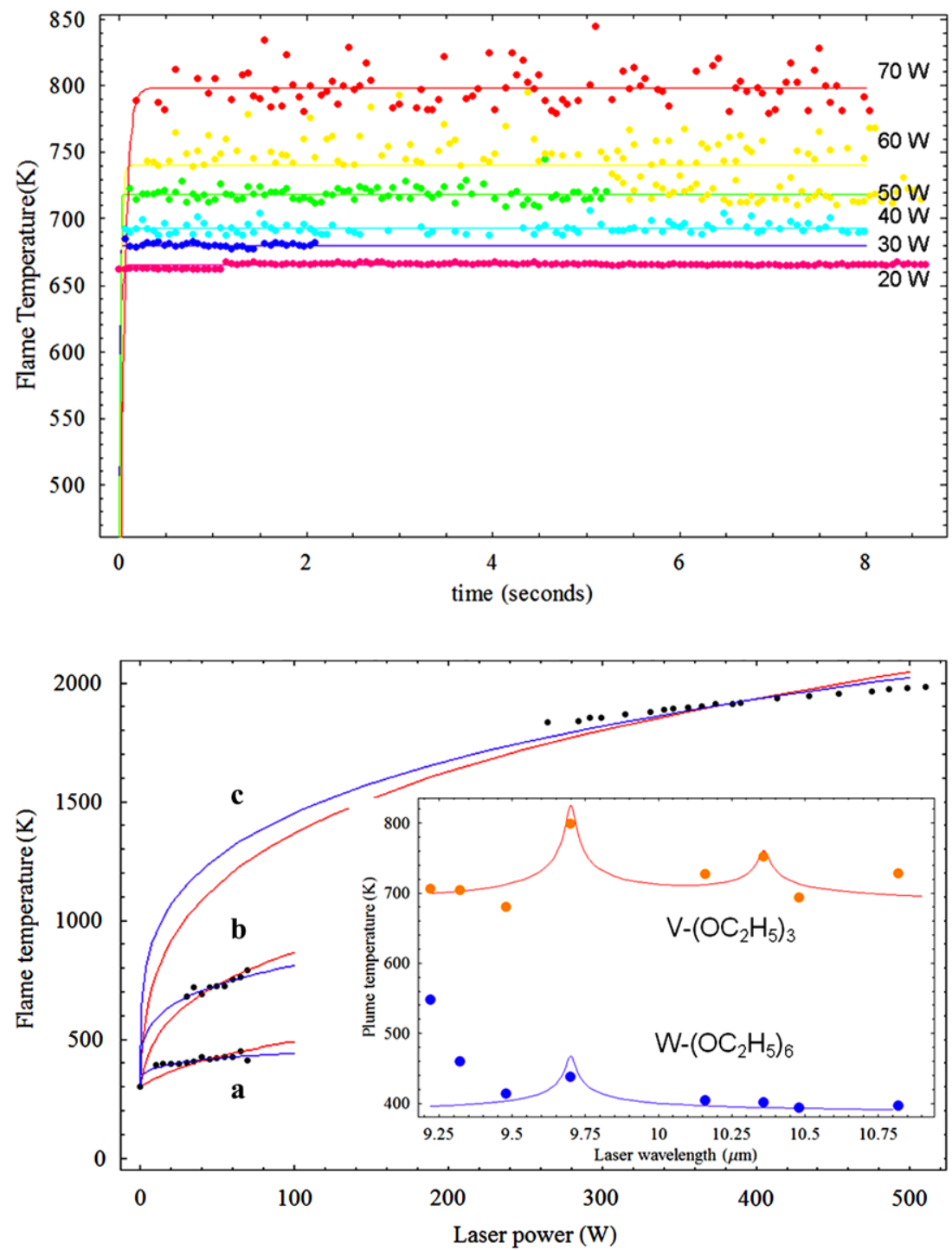

Table 1 Summary of the parameters $\mu, C_{p}, \Delta H$, and $\kappa_{\text {conv }}$ for the precursor liquids as obtained from fitting (10) to the experimental $T(t)$ data

\begin{tabular}{lllll}
\hline Starting material & $\begin{array}{l}\text { Fraction of the laser } \\
\text { power absorbed, } \mu\end{array}$ & $\begin{array}{l}\text { Specific heat capacity, } \\
C_{p}\left(\mathrm{~kJ} \mathrm{~kg}^{-1} \mathrm{~K}^{-1}\right)\end{array}$ & $\begin{array}{l}\text { Enthalpy change, } \\
\left.\Delta H\left(10^{5} \mathrm{~J} \mathrm{~mol}\right)^{-1}\right)\end{array}$ & $\begin{array}{l}\text { Convection coefficient, } \\
\kappa_{\text {conv }}\left(\mathrm{W} \mathrm{K}^{-1}\right)\end{array}$ \\
\hline $\mathrm{V}-\left(\mathrm{OC}_{2} \mathrm{H}_{5}\right)_{3}$ & 0.0432 & $(1.01 \pm 0.22) \times 10^{-5}$ & $-(7.03 \pm 1.02)$ & 0.0034 \\
& & & {$[-6.3 \mathrm{eV} / \mathrm{mol}]$} & 0.0052 \\
$\mathrm{~W}-\left(\mathrm{OC}_{2} \mathrm{H}_{5}\right)_{6}$ & 0.0133 & $(0.25 \pm 0.01) \times 10^{-5}$ & $-(8.42 \pm 1.22)$ & - \\
Benzene & 0.08 & $1.8 \pm 0.1[22]$ & $-2.08[25]$ & {$\left[-1.9 \mathrm{eV} \mathrm{mol} \mathrm{mol}^{-1}\right]$} \\
\hline
\end{tabular}

-which range from 1 to $3 \mathrm{~kJ} \mathrm{~mol}^{-1} \mathrm{~K}^{-1}$, the specific heat capacity for the laser excited $\mathrm{V}-\left(\mathrm{OC}_{2} \mathrm{H}_{5}\right)_{3}$ and $\mathrm{W}-\left(\mathrm{OC}_{2} \mathrm{H}_{5}\right)_{6}$ are found to be unusually very small of the order of $10^{-5}$ $\mathrm{kJ} \mathrm{mol}^{-1} \mathrm{~K}^{-1}$ or less. These new values are compatible with electron specific heat capacity factor which is given $[27$, 28] as $\gamma_{\mathrm{el}}=C_{\mathrm{el}} / T=\left(\pi^{2} k_{B}^{2} / 3\right) N\left(\varepsilon_{\mathrm{dos}}\right)$ where $N\left(\varepsilon_{\mathrm{dos}}\right)$ is the density of states at the Fermi level. The typical values of this factor for electrons in a vanadium atom [29] is about $20 \times 10^{-4} \mathrm{~J} \mathrm{~mol}^{-1} \mathrm{~K}^{-1}$ (Fig. 7).

The enthalpy heat of formation $\Delta H$ for vanadium oxides ( $\mathrm{VO}, \mathrm{V}_{2} \mathrm{O}_{3}, \mathrm{~V}_{2} \mathrm{O}_{5}$, and $\mathrm{VO}_{2}$ ) from their starting materials as determined from computational studies and from 
Table 2 Summary of the parameters $I_{\text {laser }}^{\text {sat }}, \lambda_{\text {res }}, \eta_{\text {res }}$ and $\kappa_{\text {conv }}$ for the precursor liquids as obtained from fitting $(25)$ to the experimental $T\left(P_{\text {laser }}\right)$ and $T\left(\lambda_{\text {laser }}\right)$ data

\begin{tabular}{llll}
\hline Starting material & $\begin{array}{l}\text { Saturation laser } \\
\text { intensity, } I_{\text {laser }}^{\text {sat }}\left(\mathrm{W} / \mathrm{m}^{2}\right)\end{array}$ & $\begin{array}{l}\text { Resonant wave lengths } \\
\lambda_{\text {res }}(\mu \mathrm{m})\end{array}$ & $\begin{array}{l}\text { Damping coefficient of } \\
\text { the bonds involved, } \eta\end{array}$ \\
\hline $\mathrm{V}-\left(\mathrm{OC}_{2} \mathrm{H}_{5}\right)_{3}$ & 2285.16 & $9.70,10.375$ & $1.6 \times 10^{3} \mathrm{~s}^{-1}$ \\
$\mathrm{~W}-\left(\mathrm{OC}_{2} \mathrm{H}_{5}\right)_{6}$ & 1104.87 & 9.70 & $1.3 \times 10^{3} \mathrm{~s}^{-1}$ \\
\hline
\end{tabular}

empirical tables are in the range -6 to $-8 \mathrm{eV} / \mathrm{mol}$ [29]. The fitting of (6) to our present experimental temperaturetime data yielded a heat of formation of $\Delta H \sim-(7 \pm$ 1) $\times 10^{5} \mathrm{~J} \mathrm{~mol}^{-1}$ which is $-(6.25 \pm 1.04) \mathrm{eV} \mathrm{mol}^{-1}$. Similarly, for $\Delta H$ for tungsten oxides, especially $\mathrm{WO}_{3}$, is $-842.87 \mathrm{~kJ} \mathrm{~mol}^{-1}\left(-8.4287 \times 105 \mathrm{~J} \mathrm{~mol}^{-1}\right)$ which is -8.78 $\mathrm{eV} \mathrm{mol}^{-1}[29]$. Our current values for $\Delta H, \mu, C_{p}$, and $\kappa_{\mathrm{conv}}$ are listed in Table 1. This is clear evidence that laser heating of the vanadium and tungsten containing ethoxides interacts with electrons rather than with the bulk materials in agreement with previous observations with $\mathrm{KrF}-$-excimer laser studies [30-33].

\subsection{Laser power and wavelength effects on temperature: saturation and damping}

A second part of the flame temperature data shows the variation with laser power when the wavelength is kept constant at $10.6 \mu \mathrm{m}$ as well as its variation with changing laser wavelength at a constant laser power of $30 \mathrm{~W}$. The plots for flame temperature versus laser power and the fitting of (14) and (15) to the experimental data are illustrated in Fig. 5(a) for $\mathrm{W}-\left(\mathrm{OC}_{2} \mathrm{H}_{5}\right)_{6}, 5(\mathrm{~b}) \mathrm{V}-\left(\mathrm{OC}_{2} \mathrm{H}_{5}\right)_{3}$. Our data plots have similar trends to previous findings by Tenegal et al. in the laser pyrolysis of benzene as shown in Fig. 5(c).

The fittings enable the determination of damping coefficients and hence saturation intensities for the materials as shown in (11) above. For the case of varying wavelength at a constant laser power, the $\mathrm{CO}_{2}$ laser power spectrum allows no more than 10 wavelength points in the wavelength range 9.22 to $11.26 \mu \mathrm{m}$. Due to low power, our laser gives only eight $\left(T, \lambda_{\text {laser }}\right)$ points of these which have been plotted in the inset of Fig. 5 for the two starting liquids. In this inset, (15) has been fitted taking $P_{\text {laser }}=30 \mathrm{~W}$ and then resonant wavelengths are extracted. These results been summarized in Table 2.

The vanadium containing precursor has a larger damping coefficient (or faster relaxation rate) and hence a larger saturation intensity than the tungsten containing one. This means that the vanadium containing liquid is harder to dissociate into its final product than that of tungsten. These results corroborate the higher enthalpies for $\mathrm{V}-\left(\mathrm{OC}_{2} \mathrm{H}_{5}\right)_{3}$ than $\mathrm{W}-\left(\mathrm{OC}_{2} \mathrm{H}_{5}\right)_{6}$ discussed before and shown in Table 1 and the usual experience that the $\mathrm{VO}_{2}$ is more difficult to obtain than $\mathrm{WO}_{3}$, for instance.

\section{Conclusion}

We have observed flames that were invisible to the naked eye at the interaction zone between the laser beam and the tungsten ethoxide aerosol flow by means of an infrared camera. Radiance of such flames at varying laser power was determined by comparison with a blackbody source of known emissivity (unity) and known temperature. Emissivities of the precursor solutions were determined from their FTIR spectra by comparing these to the blackbody radiation spectrum.

Spectral emissivity of the flame was determined from the pump $\left(\mathrm{CO}_{2}\right.$ laser) - probe (blackbody source) technique and determining transmittance of the flame to the blackbody radiation. In order to explain the temperature-laser power and temperature-wavelength data, we have derived two equations: (1) based on the extension of the Haggerty-Cannon energy balance equation in laser pyrolysis where the relation of flame temperature as a function of time and laser power is obtained and (2) imposed the standard forced oscillator differential equation in the energy balance to obtain temperature as a function of both laser power and laser wavelengths. An equation derived from the Haggerty and Cannon energy balance model has been rederived and fitted to the vanadium and tungsten ethoxide data.

From the present model, it has been found that only a small amount of laser power is used to transform the precursors into the desired nanostructures- $1.33 \%$ for $\mathrm{W}-$ $\left(\mathrm{OC}_{2} \mathrm{H}_{5}\right)_{6}, 4.32 \%$ for $\mathrm{V}-\left(\mathrm{OC}_{2} \mathrm{H}_{5}\right)_{3}$ and $8 \%$ for benzene. The $1.33 \%$ and $4.32 \%$ values are in good agreement with the ratio of the laser power measured in our lab before and after the interaction at different laser power input values. Enthalpies of formation have also been determined by both the present theories and experiment with a high degree of agreement.

However, specific heat capacities are much lower than those expected for the solvent ethanol that is used to dissolve the present precursor liquids. It has been discussed that the values of the specific heat capacities obtained here are not bulk capacities but rather agree very well with electronic heat capacities. This agrees with the known fact that laser photons interact with charged fundamental particles in the precursor materials which are largely the free electrons.

The latter theory and experimental data sets on temperature-power and temperature-wavelength have shown than 
the vanadium containing liquid is harder to dissociate into its final products than the tungsten one. It can be concluded that lighter molecules tend to dissociate at higher energies that heavier ones, this being in keeping with resonance condition $\omega_{\text {resonance }}^{2}=\left(k / \mu^{*}\right)$ where, for the same elastic constant, $k$, the bigger the reduced mass $\mu^{*}$, the lower the resonance frequency and hence the lower the energy of dissociation.

Acknowledgements The assistance during the experiments from Malcolm Govender, Lerato Shikwambana, Bathusile Masina, and Steven Nkosi is appreciated. Professor Elias Sideras-Haddad is acknowledged for the laser pyrolysis chamber and Professor Andrew Forbes for the useful discussion. Sponsorship for this project came from the CSIR parliamentary grants (PG): LHIE100 (Advanced Photonic Materials), LHTE300 (User Facility), and the Southern Education and Research Alliance (SERA) of the CSIR-University of Pretoria agreement under project number LHTAF00/07000/07020/REV.

Open Access This article is distributed under the terms of the Creative Commons Attribution Noncommercial License which permits any noncommercial use, distribution, and reproduction in any medium, provided the original author(s) and source are credited.

\section{References}

1. A. Kaldor, R.L. Woodin, Proc. IEEE 70, 565 (1982)

2. E.F. Nichols, G.F. Hull, Phys. Rev. 13, 307 (1901)

3. Crookes radiometer demonstration from the American Association of Physics Teachers

4. D.J. Wineland, W.M. Itano, Phys. Today, June (1987)

5. H.R. Bachmann, H. Noth, R. Rinck, K.S. Kompa, Chem. Phys. Lett. 29, 627 (1975)

6. B.W. Mwakikunga, A. Forbes, E. Sideras-Haddad, R.M. Erasmus, G. Katumba, B. Masina, Int. J. Nanopar. 1, 3 (2008)

7. B.W. Mwakikunga, A. Forbes, E. Sideras-Haddad, C. Arendse, Phys. Status Solidi A 205, 150 (2008)

8. B.W. Mwakikunga, A. Forbes, E. Sideras-Haddad, C. Arendse, Nanoscale Res. Lett. 3, 372 (2008)

9. B.W. Mwakikunga, E. Sideras-Haddad, C. Arendse, J. Nanosci, Nanotechnol. 9, 3286 (2009)
10. C.M. Bowden, J.D. Stettler, N.M. Witriol, J. Phys., B At. Mol. Opt. Phys. 34, 1045 (1977)

11. J.S. Haggerty, W.R. Cannon, in ed by J.L. Steinfield Laser Induced Chemical Processes (Plenum Press, New York, 1981) p. 165

12. F. Tenegal, S. Petcu, N. Herlin-Boime, X. Armand, M. Mayne, C. Reynaud, Chem. Phys. Lett. 335, 155 (2001)

13. F. El-Diasty, Opt. Commun. 241, 121 (2004)

14. O. Bomatí-Miguel, X.Q. Zhao, S. Martelli, P.E. Di Nunzio, S. Veintemillas-Verdaguer, J. Appl. Phys. 107, 014906 (2010)

15. R.W. Cannon, C.S. Danforth, J.H. Flint, S.J. Haggerty, A.R. Marra, J. Am. Ceram. Soc. 65, 324 (1982)

16. R.W. Cannon, C.S. Danforth, S.J. Haggerty, A.R. Marra, J. Am. Ceram. Soc. 65, 335 (1982)

17. V.V. Afanas'ev, S.A. Abrukov, N.I. Kidin, A.K. Kuz'min, Combust. Explos. Shock Waves 31, 432 (1995)

18. V.P. Samsonov, Combust. Explos. Shock Waves 39, 270 (2003)

19. P. Mehta, A. Sarma, J. Ghosh, S. Pandya, S. Pandya, P. Choudhuri, J. Govindarajan, C.I. Schrittwieser, R. Schrittwieser, Phys. Scr. 82, 055402 (2010)

20. C.T. O’Sullivan, Am. J. Phys. 58, 956 (1990)

21. P. Gould, Am. J. Phys. 65, 1120 (1997)

22. S. Chu, L. Hollberg, J.E. Bjorkholm, A. Cable, A. Ashkin, Phys. Rev. Lett. 55, 48 (1985)

23. B.W. Mwakikunga, A. Forbes, E. Sideras-Haddad, M. Scriba, E. Manikandan, Nanoscale Res. Lett. 5, 389 (2010)

24. J. Livage, Chem. Mater. 3, 578 (1991)

25. B.W. Mwakikunga, E. Sideras-Haddad, M. Maaza, Opt. Mater. 29, 481 (2007)

26. C.J. Willers, private communication

27. J. Forster, Th. Hagen, M. von Hoesslin, J. Uhlebusch, Appl. Phys. B 62, 263 (1996). http://www.engineeringtoolbox.om/specificheat-fluids-d_151.html

28. A.S. Neshpor, G.V. Samsonov, Consultants Bureau (Plenum, New York, 1972) pp. 1121-1124 [Kiev Polytechnical Insitute, Translated from Izvestiya Vyeshikh Uchebnykh Zavedenii, Fisika, No. 9, 23-27 (1969)]

29. Z. Lin, L.V. Zhigilei, V. Celli, Phys. Rev. B 77, 075133 (2008)

30. L. Wang, T. Maxisch, G. Ceder, Phys. Rev. B, Condens. Matter Mater. Phys. 73, 195107 (2006)

31. C. Xian-e, D. Daichun, N. Jianping, Z. Jing, Q. Yixiang, Thermochim. Acta 292, 45 (1997)

32. H. Willwohl, J. Wolfrum, Appl. Surf. Sci. 54, 89 (1992)

33. A. Arnold, B. Lange, T. Bouché, T. Heitzmann, G. Schiff, W. Ketterle, P. Monkhouse, J. Wolfram, Ber. Bunsenges. Phys. Chem. 96, 1388 (1992) 